\title{
Proprotein convertase subtilisin/kexin type 9 inhibition in cardiovascular disease: current status and future perspectives
}

\author{
Kyung Hoon Cho and Young Joon Hong
}

Division of Cardiology, Chonnam National University Medical School, Gwangju, Korea

\author{
Received: April 7, 2020 \\ Accepted: June 20, 2020 \\ Correspondence to \\ Young Joon Hong, M.D. \\ Division of Cardiology, \\ Chonnam National University \\ Hospital, 42 Jebong-ro, \\ Dong-gu, Gwangju 61469, \\ Korea \\ Tel: $+82-62-220-5778$ \\ Fax: +82-62-223-3105 \\ E-mail: hyj20o@hanmail.net \\ https://orcid.org/0000-0003- \\ o192-8161
}

Proprotein convertase subtilisin/kexin type 9 (PCSK9) targets the degradation of low-density lipoprotein (LDL) receptors; it has been proved that its inhibition improves cardiovascular outcomes in patients with established atherosclerotic cardiovascular disease (ASCVD). Herein, we review the current status of PCSK9 inhibitors in clinical practice and the future scope of $\mathrm{PCSK}_{9}$ inhibition. The results of two recent large clinical trials reveal that two PCSK9 monoclonal antibodies evolocumab and alirocumab reduce the risk of a cardiovascular event on top of background statin therapy in patients with stable ASCVD and those with recent acute coronary syndrome, respectively. However, there are several ongoing concerns regarding the efficacy in reducing mortality, cost-effectiveness, and long-term safety of extremely low LDL cholesterol levels with PCSK9 inhibition. The results of ongoing cardiovascular outcomes trials with PCSK9 monoclonal antibodies for primary prevention and with small interfering RNA to PCSK9 for secondary prevention may help to shape the use of this new therapeutic class.

Keywords: PCSK9 protein, human; Cardiovascular diseases; Antibodies, monoclonal; Cholesterol, LDL

\section{INTRODUCTION}

Atherosclerotic cardiovascular disease (ASCVD) is the leading cause of death worldwide [1]. Because the estimated growth of the aging population offsets the benefits achieved by the improved treatment and the mitigation of the risk factors of ASCVD, a sustained and high global mortality rate due to it could be evident by 2030 [2]. Multiple lines of evidence from genetic, epidemiologic, and clinical studies have converged on plasma cholesterol, particularly low-density lipoprotein (LDL) cholesterol, as the principal cause of the initiation and progression of ASCVD [3]. Although statins (3-hydroxy-3-methylglutaryl-coenzyme A reductase inhibitors) have been the primary treatment of choice for treating dyslipidemia for decades, the residual risk for high-risk patients remains high despite statin therapy $[4,5]$. Furthermore, adverse effects such as statin-associated muscle symptoms and diabetes mellitus limit their use in high-risk patient populations [6].

The discovery of proprotein convertase subtilisin/kexin type 9 ( $\left.\mathrm{PCSK}_{9}\right)$ in 2003 has rapidly transformed our understanding of lipoprotein metabolism [7]. PCSK9 diminishes the clearance of LDL cholesterol from the circulation by promoting the degradation of LDL receptors, and the results of two recent cardiovascular outcomes trials indicate that the incremental benefit of adding a PCSK9 inhibitor on top of statin therapy in patients with ASCVD [8-10]. Two PCSK9 monoclonal antibodies, evolocumab and alirocumab, received U.S. 
Food and Drug Administration (FDA) approval in 2015. Alirocumab was approved to treat heterozygous familial hypercholesterolemia or individuals with clinical ASCVD who require further LDL cholesterol-lowering in combination with other treatments typically including a maximally tolerated statin. Evolocumab was approved for patients with clinical ASCVD or heterozygous or homozygous familial hypercholesterolemia who require further LDL cholesterol-lowering in combination with other lipid-lowering treatments. In this review, we discuss the current role of PCSK9 inhibitors in clinical practice and how $\mathrm{PCSK}_{9}$ inhibition may be expanded beyond its current position based on recent and emerging evidence.

\section{RATIONALE AND STRATEGIES FOR PCSK9 INHIBITION}

PCSK9 is a member of the proteinase K subfamily of subtilisin-related serine endoproteases. The physiologic function of PCSK9 was initially discovered by the identification of point mutations in PCSKg that cause an autosomal dominant form of hypercholesterolemia [7]. PCSK9, like LDL cholesterol, serves as a ligand for LDL receptors. After PCSK9 binds to the LDL receptors on the hepatocyte surface, it targets the receptors for lysosomal degradation. Thus, PCSK9 promotes LDL receptor degradation, thereby consequentially decreasing the clearance of LDL cholesterol from the circulatory system [8]. Studies have shown that the gain or loss of function by mutations in PCSK9 cause higher or lower level of LDL cholesterol, respectively, which is associated with a corresponding higher or lower risk of coronary heart disease $[7,8]$. Moreover, statins upregulate the expression and secretion of PCSK9 by activating sterol regulatory element-binding protein-2, which increases the transcription of both LDL receptors and PCSK9 mRNA [11]. Consequently, PCSK9 inhibition seems to be a very attractive strategy for lowering LDL cholesterol and enhancing the efficacy of statin therapy.

Several therapeutic approaches to the inhibition of PCSK9 have been proposed (Fig. 1). Monoclonal antibodies for PCSK9 have been developed and two of them (evolocumab and alirocumab) have proved to be effective in reducing cardiovascular outcomes on top of background statin therapy in patients with ASCVD $[9,10]$. Furthermore, small interfering ribonucleic acid (siRNA) affects intracellular hepatocyte PCSK9 production through the targeted inhibition of translation [12]. Inclisiran, the most promising siRNA, is currently being studied in a dedicated phase III cardiovascular outcomes trial (ORION-4, NCT03705234). Another approach to $\mathrm{PCSK}_{9}$ inhibition is through vaccination to incite active immunization by producing long-lasting anti-PCSK9 antibodies. The only vaccine against PCSK9 is currently undergoing a phase I clinical trial (NCTo2508896) [13].

\section{EFFICACY OF PCSKg INHIBITORS}

\section{LDL cholesterol-lowering effects}

The LDL cholesterol-lowering effects of both alirocumab and evolocumab have been described in several phase III clinical trials involving patients with familial hypercholesterolemia and mixed dyslipidemia treated with other lipid-lowering therapies. Evolocumab reduced LDL cholesterol by $18.3 \%$ to $66 \%$ over 12 to 52 weeks [14-22], while the reduction by alirocumab was $36.3 \%$ to $61.0 \%$ over 24 to 78 weeks [23-33]. The findings of two recent large clinical outcomes trials described the profound LDL cholesterol reduction by PCSK9 inhibitors [9,10]. Further Cardiovascular Outcomes Research with PCSK9 Inhibition in Subjects with Elevated Risk (FOURIER) trial involving 27,564 patients on statin therapy with ASCVD and LDL cholesterol level $\geq 70 \mathrm{mg} / \mathrm{dL}$ disclosed that evolocumab reduced LDL cholesterol by $59 \%$ on average compared with the placebo group after 48 weeks from a median baseline value of $92 \mathrm{mg} / \mathrm{dL}(2.4 \mathrm{mmol} / \mathrm{L})$ to $30 \mathrm{mg} / \mathrm{dL}$ (0.78 mmol/L) [9]. The results of the Evaluation of Cardiovascular Outcomes After an Acute Coronary Syndrome During Treatment With Alirocumab (ODYSSEY OUTCOMES) trial involving 18,924 patients with acute coronary syndrome (ACS) 1 to 12 months earlier who had an LDL cholesterol level $\geq 70 \mathrm{mg} / \mathrm{dL}$ ( 1.8 $\mathrm{mmol} / \mathrm{L})$, non-high-density lipoprotein cholesterol level $\geq 100 \mathrm{mg} / \mathrm{dL}$ ( $2.6 \mathrm{mmol} / \mathrm{L})$, or apolipoprotein B level $\geq$ $80 \mathrm{mg} / \mathrm{dL}$ revealed that alirocumab reduced LDL cholesterol by $61.0 \%$ on average compared with the placebo group at 48 weeks from a mean baseline level of $92 \mathrm{mg} /$ dL (2.4 mmol/L) to $42 \mathrm{mg} / \mathrm{dL}$ (1.1 mmol/L) [10]. Qamar et al. [34] demonstrated minimal inter-individual variabil- 


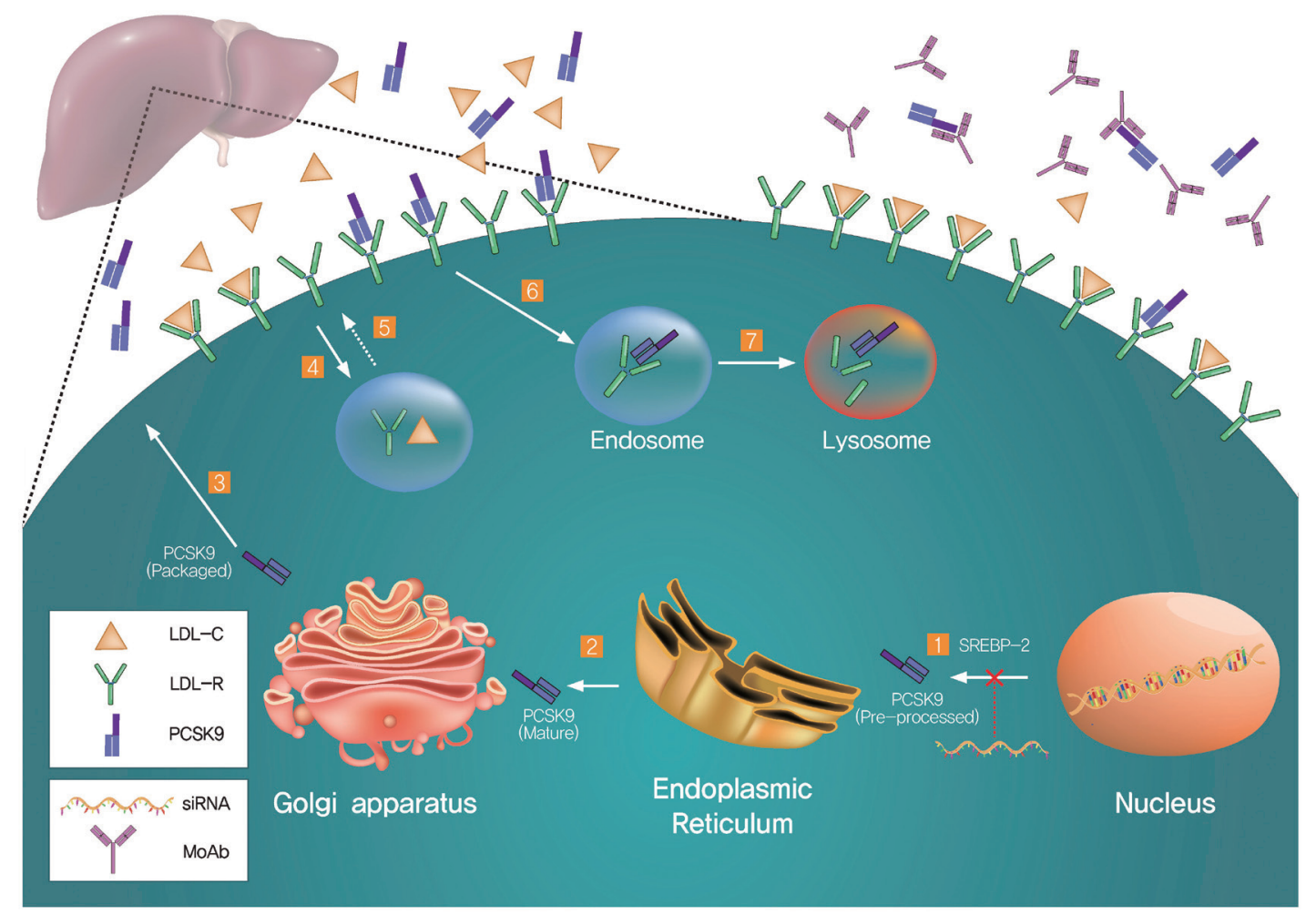

Figure 1. Proprotein convertase subtilisin/kexin type 9 (PCSK9)-mediated regulation of low-density lipoprotein receptors (LDL-Rs) and representative targets for therapeutics. (1) Sterol regulatory element-binding protein-2 (SREBP-2) increases the transcription of both LDL-Rs and PCSK9. PCSK9 is processed in the endoplasmic reticulum (2) and packaged in the Golgi apparatus (3) before being secreted. The LDL-Rs bind LDL cholesterol (LDL-C) on the cell surface, and the complex is internalized by the cells via the endosome (4). LDL-C is displaced from the LDL-Rs for use elsewhere and the LDL-Rs are recycled to the cell surface (5). Secreted PCSK9 binds to the LDL-Rs and the complex enters into the endosome (6), resulting in the lysosomal degradation of the LDL-Rs (7). There are two representative approaches to PCSK9 inhibition: small interfering ribonucleic acids (siRNAs) and PCSK9 monoclonal antibodies (MoAbs). siRNAs inhibit the translation of PCSK9 (right lower area), while MoAbs block PCSK9 binding to the LDL-Rs on the cell surface (right upper area).

ity in LDL cholesterol-lowering with $90.5 \%$ of patients achieving LDL cholesterol reduction by at least 50\% and $99.8 \%$ by at least $30 \%$ in the FOURIER trial. The results of a large meta-analysis of 35 randomized controlled trials (RCTs) involving 45,539 patients divulged significant LDL cholesterol reduction after treatment with PCSK9 inhibitors of $-60.94 \%$ and $-31.32 \%$ versus the placebo and ezetimibe groups, respectively [35]. Treatment with the PCSK9 inhibitor was associated with a favorable change in total cholesterol (mean difference [MD], -34.95; 95\% confidence interval [CI], -37.53 to $-32.37 ; p<0.001$ ), high-density lipoprotein cholesterol (MD, 6.85; 95\% CI, 6.10 to $7.60 ; p<0.001$ ), lipoprotein(a) (Lp(a); MD, -26.45; 95\% CI, -28.88 to $-24.03 ; p<0.001)$, and apolipoprotein B (MD, $-45.50 ; 95 \% \mathrm{CI},-48.35$ to $-42.64 ; p<0.001$ ).

\section{Effects on the risk of a cardiovascular event}

The FOURIER and ODYSSEY OUTCOMES trials were two pivotal RCT designed to evaluate whether PCSK9 monoclonal antibodies evolocumab and alirocumab can reduce the risk of a cardiovascular event on a background of statin therapy in patients with ASCVD (Table 1). The FOURIER trial included 27,564 patients with clinically evident ASCVD (a history of myocardial infarction, non-hemorrhagic stroke, or symptomatic peripheral artery disease) and a lipid profile characterized by an LDL cholesterol level $\geq 70 \mathrm{mg} / \mathrm{dL}(1.8 \mathrm{mmol} / \mathrm{L})$ or a non-high-density lipoprotein cholesterol levels $\geq 100$ $\mathrm{mg} / \mathrm{dL}(2.6 \mathrm{mmol} / \mathrm{L})$ despite maximal tolerated statins and/or ezetimibe [9]. They were randomly assigned in a 1:1 ratio to receive subcutaneous injections of evolocum$\mathrm{ab}$ (either $140 \mathrm{mg}$ every 2 weeks or $420 \mathrm{mg}$ monthly) or 
Table 1. Comparison of the FOURIER and ODYSSEY OUTCOMES clinical trials

\begin{tabular}{|c|c|c|}
\hline Clinical trial & FOURIER [9] & ODYSSEY OUTCOMES [10] \\
\hline Design & $\begin{array}{l}\text { Randomized, double-blinded, placebo- } \\
\text { controlled }\end{array}$ & $\begin{array}{l}\text { Randomized, double-blinded, placebo- } \\
\text { controlled }\end{array}$ \\
\hline Intervention & $\begin{array}{l}\text { Evolocumab (either } 140 \mathrm{mg} \text { every } 2 \text { wk or } \\
420 \mathrm{mg} \text { monthly) }\end{array}$ & $\begin{array}{l}\text { Alirocumab ( } 75 \text { mg every } 2 \text { wk and } \\
\text { adjusted to target an LDL-C of } 25 \text { to } 50 \\
\mathrm{mg} / \mathrm{dL})^{\mathrm{a}}\end{array}$ \\
\hline Number of patients & 27,564 (mean age $63 \mathrm{yr}$, female $25 \%$ ) & 18,924 (mean age 58 yr, female $25 \%$ ) \\
\hline Population & $\begin{array}{l}\text { Stable ACVD and LDL-C } \geq 70 \mathrm{mg} / \mathrm{dL} \\
\text { despite statin therapy }\end{array}$ & $\begin{array}{l}\text { ACS in the previous } 1 \text { to } 12 \text { mon and } \\
\text { LDL-C } \geq 70 \mathrm{mg} / \mathrm{dL} \text { despite statin } \\
\text { therapy }\end{array}$ \\
\hline Patients on high-intensity therapy, \% & $69 \cdot 3$ & 88.8 \\
\hline Follow-up duration, median yr & 2.2 & 2.8 \\
\hline Median baseline LDL-C, mg/dL & 92 & 87 \\
\hline $\begin{array}{l}\text { Mean percentage reduction in LDL-C } \\
\text { compared to the placebo group }\end{array}$ & $59 \%$ at $48 \mathrm{wk}$ & $61 \%$ at 12 mon \\
\hline Primary end point & $\begin{array}{l}\text { A composite of cardiovascular death, } \\
\text { myocardial infarction, stroke, } \\
\text { hospitalization for unstable angina, or } \\
\text { coronary revascularization }\end{array}$ & $\begin{array}{l}\text { A composite of death from coronary } \\
\text { heart disease, nonfatal myocardial } \\
\text { infarction, fatal or nonfatal ischemic } \\
\text { stroke, or unstable angina requiring } \\
\text { hospitalization }\end{array}$ \\
\hline HR for primary endpoint, $95 \%$ CI & $0.85(0.79-0.92)$ & $0.85(0.78-0.93)$ \\
\hline HR for all-cause mortality, 95\% CI & $1.04(0.91-1.19)$ & $0.85(0.73-0.98)$ \\
\hline
\end{tabular}

FOURIER, Further Cardiovascular Outcomes Research with PCSK9 Inhibition in Subjects with Elevated Risk; ODYSSEY OUTCOMES, Evaluation of Cardiovascular Outcomes After an Acute Coronary Syndrome During Treatment With Alirocumab; LDL-C, low-density lipoprotein cholesterol; ACVD, atherosclerotic cardiovascular disease; ACS, acute coronary syndrome; $\mathrm{HR}$, hazard ratio; CI, confidence interval.

${ }^{a}$ Alirocumab was held for sustained LDL-C levels below $15 \mathrm{mg} / \mathrm{dL}$.

the placebo. The results of this study showed that evolocumab significantly reduced the risk of the primary endpoint (a composite of cardiovascular death, myocardial infarction, stroke, hospitalization for unstable angina, or coronary revascularization; 1,344 patients [9.8\%] vs. 1,563 patients [11.3\%]; hazard ratio [HR], 0.85; 95\% CI, 0.79 to $0.92 ; p<0.001$ ) at 2.2 years. Evolocumab significantly reduced the risk of different types of myocardial infarction: myocardial infarction by $27 \%$ (468 patients [3.4\%] vs. 639 patients [4.6\%]; HR, 0.73; 95\% CI, 0.65 to o.82; $p<$ ०.001); first myocardial infarction by $27 \%$ (4.4\% vs. $6.3 \%$; HR, 0.73; $95 \%$ CI, 0.65 to o.82; $p<0.001$ ); type 1 by $32 \%$ (2.9\% vs. $4.5 \%$; HR, $0.68 ; 95 \% \mathrm{CI}, 0.59$ to 0.79 ; $p<0.001$ ); and type 4 by $35 \%$ (o.8\% vs. 1.1\%; HR, o.65; $95 \%$ CI, 0.48 to $0.87 ; p=0.004)$, with no effect on type 2 (0.9\% vs. $0.8 \%$; HR, 1.09; 95\% CI, 0.82 to $1.45 ; p=0.56$ ) [36]. Evolocumab significantly reduced the risk of all types of stroke (207 patients [1.5\%] vs. 262 patients [1.9\%]; HR, 0.79; 95\% CI, 0.66 to $0.95 ; p=0.01)$ and of ischemic stroke $(1.2 \%$ vs. 1.6\%; HR, $0.75 ; 95 \% \mathrm{CI}, 0.62$ to $0.92 ; p=0.005)$, with no effect on hemorrhagic stroke (0.21\% vs. $0.18 \%$; HR, 1.16; 95\% CI, 0.68 to 1.98; $p=0.59$ ) [37].

The ODYSSEY OUTCOMES trial included 18,924 patients with ACS 1 to 12 months earlier and a lipid profile characterized by a LDL cholesterol level $\geq 70 \mathrm{mg} / \mathrm{dL}$ (1.8 mmol/L), a non-high-density lipoprotein cholesterol level $\geq 100 \mathrm{mg} / \mathrm{dL}$ ( $2.6 \mathrm{mmol} / \mathrm{L})$, or apolipoprotein $\mathrm{B} \geq$ $80 \mathrm{mg} / \mathrm{dL}$ despite maximal tolerated statin [10]. Patients 
were randomly assigned in a 1:1 ratio to receive alirocumab subcutaneously at a dose of $75 \mathrm{mg}$ or the placebo every 2 weeks. The dose of alirocumab was adjusted in a blinded fashion to target an LDL cholesterol level of 25 to $50 \mathrm{mg} / \mathrm{dL}$ (0.6 to $1.3 \mathrm{mmol} / \mathrm{L}$ ). Alirocumab significantly reduced the primary endpoint events (a composite of death from coronary heart disease, non-fatal myocardial infarction, fatal or non-fatal ischemic stroke, or unstable angina requiring hospitalization; 903 patients [9.5\%] vs. 1,052 patients [11.1\%]; HR, $0.85 ; 95 \%$ CI, 0.73 to $0.93 ; p<$ 0.001 ) at 2.8 years. Alirocumab significantly reduced the risk of any stroke (1.3\% vs. 1.8\%; HR, 0.72; $95 \%$ CI, 0.57 to 0.91 ) and of ischemic stroke (1.2\% vs. 1.6\%; HR, 0.73; $95 \%$ CI, 0.57 to 0.93 ), with no effect on hemorrhagic stroke (0.2\% vs. $0.2 \%$; HR, 0.83 ; $95 \%$ CI, 0.42 to 1.65 ) [38].

There were different findings regarding the effects on mortality reduction by $\mathrm{PCSK} 9$ inhibitors in the FOURIER and ODYSSEY OUTCOMES trials. In the FOURIER trial, there was no difference in cardiovascular mortality (251 patients [1.8\%] vs. 240 patients [1.7\%]; HR, 1.05; 95\% CI, 0.88 to 1.25 ) or all-cause mortality (444 patients [3.2\%] vs. 426 patients [3.1\%]; HR, 1.04; 95\% CI, 0.91 to 1.19) between the evolocumab and placebogroups [9]. In the ODYSSEY OUTCOMES trial, alirocumab significantly reduced all-cause mortality (334 patients [3.5\%] vs. 392 patients [4.1\%]; HR, $0.85 ; 95 \%$ CI, 0.73 to 0.98 ), with no effect on cardiovascular mortality (240 patients [2.5\%] vs. 271 patients [2.9\%]; HR, 0.88; 95\% CI, 0.74 to 1.05 ) [10]. This difference was probably due to the higher cardiovascular risk of patients enrolled in the ODYSSEY OUTCOMES trial compared with those in the FOURIER trial, as the ODYSSEY OUTCOMES trial included patients who had ACS within 1 year of enrollment.

A large meta-analysis of 35 RCTs comprising 45,539 patients showed that compared with no PCSK9 inhibitor therapy, treatment with a PCSK9 inhibitor was associated with a lower rate of myocardial infarction $(2.3 \%$ vs. 3.6\%; odds ratio [OR], 0.72; $95 \% \mathrm{CI}, 0.64$ to $0.81 ; p<$ 0.001), stroke (1.0\% vs. 1.4\%; OR, 0.80; $95 \%$ CI, 0.67 to $0.96 ; p=0.02)$, and coronary revascularization ( $4.2 \%$ vs. 5.8\%; OR, 0.78; 95\% CI, 0.71 to $0.86 ; p<0.001$ ) [35]. Although no significant change was observed in all-cause mortality (OR, 0.71; 95\% CI, 0.47 to 1.09; $p=0.12$ ) or cardiovascular mortality (OR, 1.01; 95\% CI, 0.85 to 1.19; $p=$ 0.95), there was a significant association between higher baseline LDL cholesterol and benefit from PCSK9 in- hibitor in all-cause mortality $(p=0.038)$. The results of a more recent meta-analysis involving 54 RCTs and 97,910 patients demonstrate that compared with the controls, PCSK9 inhibitors significantly reduced the risk of major adverse cardiovascular events (a composite of cardiovascular death, non-fatal myocardial infarction, and non-fatal stroke) by $16 \%$ (relative risk [RR], $0.84 ; 95 \% \mathrm{CI}$, 0.79 to 0.89 ), non-fatal myocardial infarction by $17 \%$ (RR, $0.83 ; 95 \%$ CI, 0.74 to 0.93 ), and any stroke by $25 \%$ (RR, 0.75; $95 \% \mathrm{CI}, 0.65$ to 0.85 ) [39].

\section{Effects on $L p(a)$}

$\mathrm{Lp}(\mathrm{a})$ consists of an LDL-like particle containing apolipoprotein(a) linked to apolipoprotein B-10o. The plasma concentration of $\mathrm{Lp}(\mathrm{a})$ is highly heritable and mainly determined by the apolipoprotein(a) gene [40]. Although the physiologic function of $\mathrm{Lp}(\mathrm{a})$ remains unclear, cumulative evidence including epidemiologic and genetic data suggests that $\mathrm{Lp}(\mathrm{a})$ is an independent risk factor for ASCVD $[40,41]$. However, the therapeutic targeting of $\mathrm{Lp}(\mathrm{a})$ has proved difficult to date and there are currently no FDA-approved drugs for the treatment of elevated $\mathrm{Lp}(\mathrm{a})$. The results of a recent meta-analysis comprising 27 RCTs with 11,864 patients revealed that a $\mathrm{PCSK}_{9}$ inhibitor was associated with $\mathrm{Lp}(\mathrm{a}$ ) reduction by $21.9 \%$ ( $95 \% \mathrm{CI},-24.3$ to -19.5) [42]. O'Donoghue et al. [43] analyzed 25,096 patients having Lp(a) levels in the FOURIER trial and demonstrated that higher levels of Lp(a) were associated with an increased risk of a cardiovascular event irrespective of LDL cholesterol. Evolocumab significantly reduced Lp(a) levels and patients with higher Lp(a) levels tended to experience greater cardiovascular benefit from $\mathrm{PCSK}_{9}$ inhibition. Moreover, evolocumab reduced the risk of coronary heart disease death, myocardial infarction, or urgent revascularization by $23 \%$ (HR, 0.77; 95\% CI, 0.67 to 0.88$)$ in patients with baseline $\mathrm{Lp}(\mathrm{a})$ above the median and by $7 \%(\mathrm{HR}, 0.93 ; 95 \% \mathrm{CI}$, 0.80 to 1.08 ; $p$ interaction = o.07) in those with Lp(a) below the median.

Although the exact mechanism of PCSK9 inhibitor-induced Lp(a) lowering remains inconclusive, recent evidence suggests that $\mathrm{PCSK}_{9}$ inhibitors reduce Lp(a) concentration by both enhancing its clearance and reducing its production [44,45]. In a study involving 63 healthy men, it was demonstrated that evolocumab monotherapy lowered $\mathrm{Lp}(\mathrm{a})$ concentration by decreasing the production of Lp(a) particles [45]. In combination with statin therapy, 
evolocumab lowered the Lp(a) concentration by accelerating the $\mathrm{Lp}(\mathrm{a})$ catabolism through the marked upregulation of LDL receptors. Recently, discordance between LDL cholesterol and Lp(a) reduction in PCSK9 inhibitor therapy has been reported, which suggests that the upregulation of LDL receptors may not be the sole mechanism for Lp(a) lowering by PCSK9 inhibitors $[46,47]$.

\section{PLEIOTROPIC EFFECTS}

Similar to the "pleiotropic" effects of statins, increasing evidence supports LDL-independent activity of PCSK9 inhibition, possibly through direct anti-inflammatory and plaque-stabilizing effects. In vitro and in vivo studies have shown that PCSK9 is secreted by various cells that participate in the atherosclerotic process such as macrophages, endothelial cells, and vascular smooth muscle cells [48-50]. Oxidized LDL upregulates the production of both inflammatory cytokines (interleukin-1 $\alpha$, interleukin- 6 , and tumor necrosis factor- $\alpha$ ) and PCSK9 in macrophages. PCSK9 inhibition suppresses the activation of nuclear factor kappa B, which is a major transcription factor regulating the induction of various inflammatory mediators, resulting in the decreased production of interleukin-1 $\alpha$, interleukin- 6 , and tumor necrosis factor- $\alpha$ [48]. PCSK9-knockout mice had significantly reduced expression of lectin-type oxidized LDL receptor-1, vascular cell adhesion molecule-1, and interleukin 1 in vascular tissues compared to that in wild type mice [51]. PCSK9 gene interference has also been associated with decreased expression of vascular inflammation regulators, such as tumor necrosis factor- $\alpha$, interleukin 1 beta, monocyte chemoattractant protein-1, toll-like receptor 4, and nuclear factor kappa B [52].

There is little evidence of the pleiotropic effects of anti-PCSK9 antibodies. In a mouse model, alirocumab reduced monocyte and T-cell recruitment and improved the atherosclerotic plaque morphology by decreasing the macrophage and necrotic core content [53]. The Effects of the PCSK9 Antibody Alirocumab on Coronary Atherosclerosis in Patients With Acute Myocardial Infarction (PACMAN-AMI) trial, a phase 3 trial investigating the effect of the alirocumab on atheroma plaque and inflammation in patients with acute myocardial infarction undergoing percutaneous coronary intervention, may shed light on the pleiotropic effects of PCSK9 inhibitors (PACMAN-AMI, NCTo3067844).

\section{SAFETY OF PCSKg INHIBITORS}

Statin therapy can have negative effects on liver function and muscle enzymes, and can modestly increase new-onset diabetes [6]. The results from some small RCTs on statin therapy suggest that statins (or the low LDL cholesterol levels from their use) could be associated with impaired cognitive function [54,55]. To date, the potential association between lipid-lowering treatments and neurocognitive disorders remains an area of debate.

The safety of the two PCSK9 inhibitors evolocumab and alirocumab has been assessed in several pooled analyses, systematic reviews, and meta-analyses [35,56,57]. The results of a large meta-analysis of 35 RCTs comprising 45,539 patients infer that treatment with $\mathrm{PCSK}_{9}$ inhibitor was not associated with neurocognitive adverse events (OR, 1.12; 95\% CI, 0.88 to $1.42 ; p=0.37$ ), myalgia (OR, 0.95; 95\% CI, 0.75 to $1.20 ; p=0.65$ ), new-onset or worsening of preexisting diabetes mellitus (OR, 1.05; $95 \% \mathrm{CI}, 0.95$ to $1.17 ; p=0.32)$, and an increase in the level of creatine kinase (OR, $0.84 ; 95 \%$ CI, 0.70 to $1.01 ; p=0.06$ ) or alanine or aspartate aminotransferase (OR, 0.96; 95\% CI, 0.82 to $1.12 ; p=0.61)$ [35].

The FOURIER study by Sabatine et al. [9] was the first RCT to provide long-term safety data for $\mathrm{PCSK}_{9}$ inhibitors. For a median of 2.2 years in 27,564 patients with clinical ASCVD, there was no significant difference between the two groups with regard to adverse events including new-onset diabetes and neurocognitive events, with exception of injection site reactions $(2.1 \%$ in the evolocumab group vs. $1.6 \%$ in the placebo group). In the Evaluating PCSK9 Binding Antibody Influence On Cognitive Health in High Cardiovascular Risk Subjects (EBBINGHAUS) study by Giugliano et al. [58] involving a subgroup of patients from the FOURIER trial, the prospective assessment of their cognitive function was conducted between the two groups using the Cambridge Neuropsychological Test Automated Battery. In a total of 1,204 patients, no significant difference was observed in objectively measured cognitive function between the two groups over a median of 19-month follow-up. From the results of the ODYSSEY OUTCOMES trial of 
alirocumab involving 18,924 patients with a recent ACS, the incidence of adverse events was similar in the two groups with the exception of injection site reactions (3.8\% in the alirocumab group vs. $2.1 \%$ in the placebo group) for a median of 2.8 years [10]. From the result of a meta-analysis of 14 RCTs of alirocumab, the overall rate of neurocognitive events was low $(<1 \%)$ and there was no significant difference between the studied groups even when stratified by age or LDL cholesterol (<25 mg/dL vs. $\geq 25 \mathrm{mg} / \mathrm{dL}$ ) [59].

Therapeutic PCSK9 monoclonal antibodies include those that are fully human (e.g., evolocumab and alirocumab) and those that are humanized (e.g., bococizumab). Bococizumab is a humanized murine-derived monoclonal antibody with a murine sequence of approximately $3 \%$ remaining in the antigen-binding complementarity determining regions and as such, are more likely to induce the development of anti-drug antibodies [6o]. In six parallel trials involving 4,300 patients, high-titer anti-drug antibodies developed in a substantial proportion of the patients who received bococizumab that led to significant attenuation of the cholesterol-lowering effect [61], a result which led to the discontinuation of the clinical development of bococizumab. On the other hand, very low rates of immunogenicity were observed in clinical trials with evolocumab and alirocumab $[9,10,62,63]$.

Vitamin E transport and steroidogenesis are closely associated with LDL cholesterol metabolism and are potentially affected by very low levels of serum LDL cholesterol. In a randomized trial involving 901 patients, absolute vitamin $\mathrm{E}$ decreased in evolocumab-treated patients from the baseline to week 52 by $16 \%$ but increased by $19 \%$ when normalized for cholesterol, while red blood cell membrane vitamin E levels did not change [64]. No significant adverse effects were observed in steroid or gonadal hormones, even at very low LDL cholesterol levels.

\section{THE ECONOMIC VALUE CONSIDERATION OF PCSKg INHIBITORS}

The poor cost-effectiveness of evolocumab and alirocumab has been a limiting factor for their widespread use since their approval in 2015. In 2017, a cost-effectiveness analysis model from the FOURIER trial was creat- ed to determine whether a PCSK9 inhibitor with statin treatment strategy was more cost-effective than a statin only strategy [65]. At the annual price of $\$ 14,300$ for a PCSK9 inhibitor, the incremental cost-effectiveness ratio of statin plus PCSK9 inhibitor therapy was $\$ 337,729$ per quality-adjusted life-year, which was more than three times the commonly accepted societal threshold of $\$ 100,000$ per quality-adjusted life-year. Thus, the price of a PCSK9 inhibitor would need to drop by $62 \%$ per year $(\$ 5,459)$. Based on the cost-effectiveness analysis model from the ODYSSEY OUTCOMES trial, when replacing ezetimibe with alirocumab on top of statin therapy, the price of alirocumab would need to drop by $86 \%$ per year from $\$ 14,560$ to $\$ 1,974$ to be considered cost-effective [66]. Several groups introduced successful practice models (a systematic team-based approach with an integrated specialty pharmacy) to facilitate appropriate patient access to PCSK9 inhibitors [67-69].

The Republic of Korea has a National Health Insurance system, and since January 2020, the insurance coverage of Repatha (Evolocumab, Amgen Manufacturing Limited, Juncos, Puerto Rico) has been extended to patients with statin intolerance, familial hypercholesterolemia, and a very high risk of future cardiovascular events (Table 2) who respond insufficiently to maximally tolerated statin and ezetimibe therapy. With the insurance by the National Health Insurance system and the use of out-of-hospital pharmacies, the patient out-ofpocket expense is approximately 1,000,000 won per year (\$814) when injected every 2 weeks. It is expected that the cost of PCSK9 inhibitors will go down as time goes by and with their more extensive use.

\section{RACE FACTORS CONCERNING PCSK INHIBI- TORS}

Race factors can influence cardiovascular risk estimation, treatment intensity, and even lipid drug use [70]. Racial differences in response to statins have been previously reported, with higher rosuvastatin plasma levels having been seen in Asian patients including Japanese, Chinese, and Asian Indians than in Caucasians [71]. The results of a meta-analysis involving 20 trials assessing statin therapy and coronary atherosclerotic plaque revealed that a $>40 \%$ reduction in LDL cholesterol re- 
Table 2. Definition of conditions pertaining to a very high risk of future ASCVD events

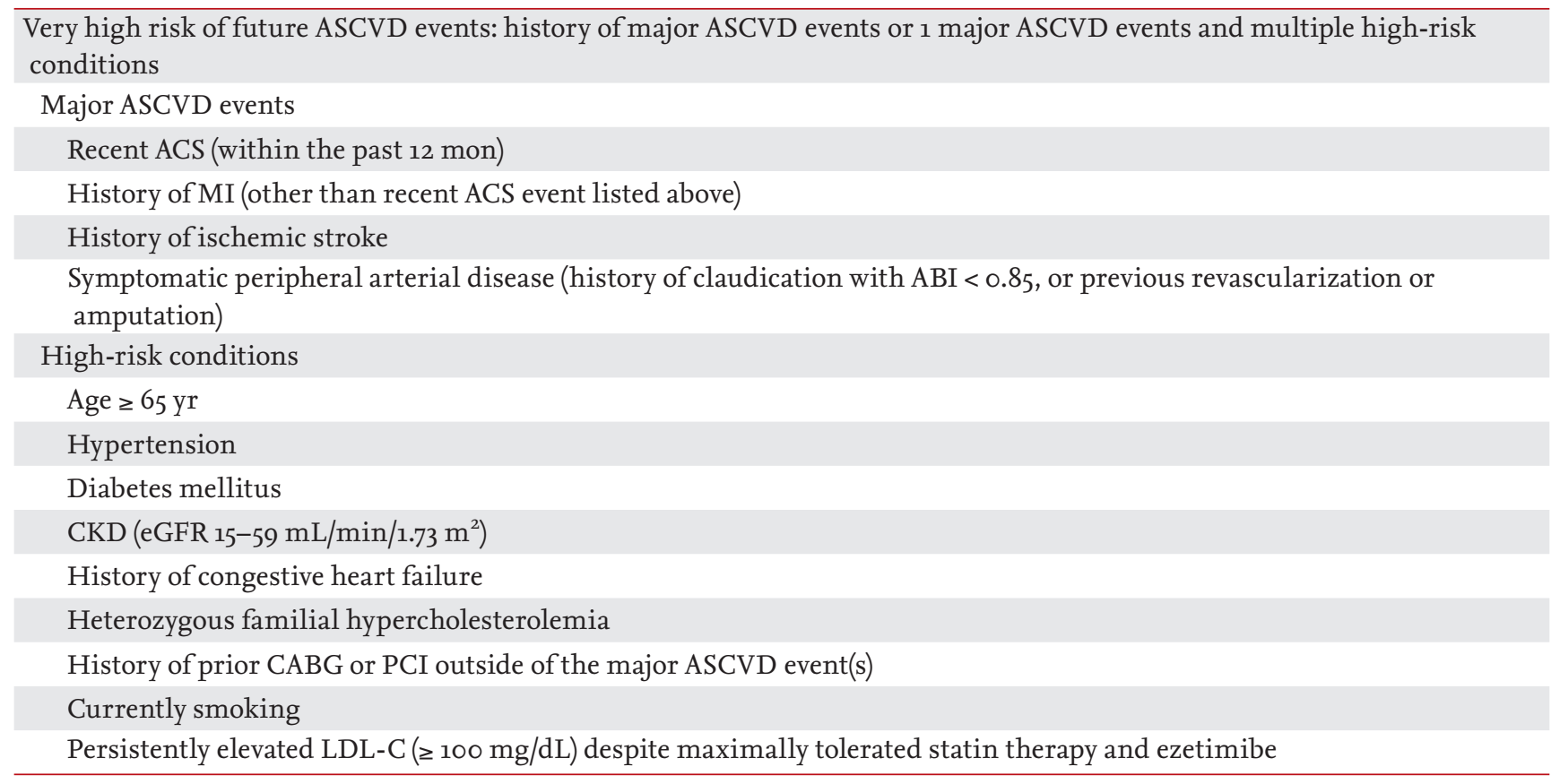

ASCVD, atherosclerotic cardiovascular disease; ACS, acute coronary syndrome; MI, myocardial infarction; ABI, ankle-brachial index; CKD, chronic kidney disease; eGFR, estimated glomerular filtration rate; CABG, coronary artery bypass graft; PCI, percutaneous coronary intervention; LDL-C, low-density lipoprotein cholesterol.

quired 80 or $40 \mathrm{mg}$ doses of atorvastatin or rosuvastatin for Westerns compared to 18.9 or 14.1 mg for Asians, respectively [72]. In this regard, FDA recommends a lower starting dose in Asian patients ( $5 \mathrm{mg}$ of rosuvastatin) compared to Caucasians (10 mg of rosuvastatin).

Wang et al. [73] evaluated the potential racial differences in the pharmacokinetics and pharmacodynamics of evolocumab in Caucasian and Asian populations; they found no significant racial differences in pharmacokinetic and pharmacodynamic relationships for the LDL cholesterol-lowering effect. In the Evaluation of Alirocumab Versus Ezetimibe on Top of Statin in Asia in High Cardiovascular Risk Patients With Hypercholesterolemia (ODYSSEY EAST) trial to assess the efficacy and safety of alirocumab versus ezetimibe in 615 Asian patients at high risk of a cardiovascular event, alirocumab significantly reduced LDL cholesterol (56\% reduction from the baseline at 24 weeks), which is consistent with the previous ODYSSEY study [74]. The results of the Randomized Trial Evaluating the Efficacy and Safety of Alirocumab in South Korea and Taiwan (ODYSSEY KT) trial involving 199 patients with hypercholesterolemia at high risk of a cardiovascular event from South Korea and Taiwan demonstrate that alirocumab changed LDL cholesterol levels by $-57.1 \%$ (placebo: $+6.3 \%$ ) at 24 weeks [75]. Alirocumab was generally well tolerated in both the ODYSSEY EAST and ODYSSEY KT trials. Although 13\% of the participants were of Asian ethnicity in the FOURIER trial and 14\% of Asia Pacific and South African ethnicity in the ODYSSEY OUTCOMES trial, more clinical data regarding the race factor on the efficacy and safety of PCSK9 inhibitors is needed $[9,10]$.

\section{GUIDELINES ON THE USE OF PCSKg INHIBITORS}

With positive clinical outcomes data on $\mathrm{PCSK}_{9}$ inhibitors including evolocumab and alirocumab, recent guidelines have updated their recommendations regarding the position of PCSK9 inhibitors for ASCVD risk reduction $[70,76,77]$. The United States guidelines released in 2018 define two patient populations with specific thresholds of LDL cholesterol for whom PCSK9 inhibitors are appropriate [70]: those with a very high risk of future cardiovascular events and LDL cholesterol $>70 \mathrm{mg} / \mathrm{dL}$ (> $1.8 \mathrm{mmol} / \mathrm{L}$ ) and those with primary hy- 
percholesterolemia (LDL cholesterol > $100 \mathrm{mg} / \mathrm{dL}$ [baseline LDL cholesterol $\geq 190 \mathrm{mg} / \mathrm{dL}$ ] or $>130 \mathrm{mg} / \mathrm{dL}$ [baseline LDL cholesterol $\geq 220 \mathrm{mg} / \mathrm{dL}]$ ) when on maximally tolerated statin and ezetimibe therapy [70]. Patients with a very high risk of a future cardiovascular event are those who have had multiple major events or one major event along with multiple high-risk conditions (Table 2). The Korean guidelines released in 2018 recommend that a PCSK9 inhibitor can be considered for very high-risk patients when the target LDL cholesterol level cannot be met even with maximally tolerated statins and/or ezetimibe or when the patient has statin intolerance [76]. In the European guidelines released in 2019, combinatory therapy with a PCSK9 inhibitor is recommended in patients at very high risk who have not achieved the target LDL cholesterol level on the maximum tolerated dose of statin and ezetimibe [77]. These guidelines emphasize the more intensive reduction of LDL cholesterol across cardiovascular event risk categories. For primary and secondary prevention in very high-risk patients with or without familial hypercholesterolemia, an LDL cholesterol reduction of $\geq 50 \%$ from the baseline and an LDL cholesterol goal of $<55 \mathrm{mg} / \mathrm{dL}(<1.4 \mathrm{mmol} / \mathrm{L})$ are recommended. For patients with ASCVD who experience a second cardiovascular event within 2 years (not necessarily of the same type as the first one) while taking maximally tolerated statins, LDL cholesterol of $<40 \mathrm{mg} /$ $\mathrm{dL}(<1.0 \mathrm{mmol} / \mathrm{L})$ is recommended as the target.

\section{FUTURE SCOPE OF PCSK 9 MONOCLONAL ANTIBODIES}

Although it has been well elucidated that PCSK9 monoclonal antibodies can reduce cardiovascular events in patients with clinical ASCVD, there are considerable areas that need to be clarified to realize the full potential of PCSK9 inhibition in the treatment of cardiovascular disease. While a reduction in all-cause mortality was observed in the ODYSSEY OUTCOMES trial in a subgroup with baseline LDL cholesterol > $100 \mathrm{mg} / \mathrm{dL}$, cardiovascular outcome studies of evolocumab and alirocumab have not shown a clear benefit on reducing mortality from coronary heart disease and cardiovascular disease $[9,10]$. Moreover, the use of PCSK9 inhibitors for the primary prevention of ASCVD is limited to patients with familial hypercholesterolemia. A phase III clinical outcomes trial to evaluate the impact of evolocumab on preventing major cardiovascular events in patients at high risk without prior myocardial infarction or stroke (Effect of Evolocumab in Patients at High Cardiovascular Risk Without Prior Myocardial Infarction or Stroke [VESALIUS-CV], NCTo3872401) is ongoing. The positive impact of high-intensity statin therapy in the early period of ACS is well established, but it is unknown whether $\mathrm{PCSK}_{9}$ inhibitors administered during the early period of ACS are effective $[78,79]$. Recently, Koskinas et al. [80] conducted a double-blind RCT to assess the feasibility, safety, and LDL cholesterol-lowering efficacy of evolocumab initiated during the in-hospital phase of ACS. A total of 308 patients hospitalized for ACS with elevated LDL cholesterol levels $(\geq 70 \mathrm{mg} / \mathrm{dL}$ on high-intensity statins for at least 4 weeks; $\geq 90 \mathrm{mg} / \mathrm{dL}$ on low- or moderate-intensity statins; or $\geq 125 \mathrm{mg} / \mathrm{dL}$ without a stable dose of statins) were randomly assigned 1:1 to receive $420 \mathrm{mg}$ of evolocumab or a matching placebo during the in-hospital period and at 4 weeks on top of $40 \mathrm{mg}$ of atorvastatin. The percentage change in LDL cholesterol from the baseline to 8 weeks was $-77.1 \% \pm 15.8 \%$ in the evolocumab group versus $-35.4 \% \pm 26.6 \%$ in the placebo group $(p<0.001)$. Adverse and centrally adjudicated cardiovascular events were similar in both groups. These results call for large clinical outcomes trials to assess whether very early LDL cholesterol-lowering with $\mathrm{PCSK}_{9}$ inhibitors on top of statin therapy is effective at reducing cardiovascular events after ACS.

\section{OTHER APPROACHES TO PCSKg INHIBITION}

The development of other approaches to PCSK9 inhibition to overcome the limitations of PCSK9 monoclonal antibodies, such as the need for frequent administration and poor cost-effectiveness, are underway. The siRNA inclisiran is the most promising non-antibody $\mathrm{PCSK}_{9}$ inhibitor. While monoclonal antibodies target extracellular PCSK9, inclisiran inhibits translation of PCSK9 protein, thereby resulting in decreased synthesis of intracellular PCSK9 in the liver [12]. In a phase II trial involving 501 patients at high risk of a cardiovascular event with elevated LDL cholesterol (> 70 and $>100 \mathrm{mg}$ / $\mathrm{dL}$ for patients with and without a history of ASCVD, 
respectively) despite maximal statin therapy, inclisiran decreased LDL cholesterol in a dose-dependent manner with reductions of up to more than $50 \%$ [81]. The inclisiran group received a single dose of 200,300 , or $500 \mathrm{mg}$ or two doses (at days 1 and 90) of 100, 200, or $300 \mathrm{mg}$. At day 180, the least-squares mean reductions in LDL cholesterol levels were $27.9 \%$ to $41.9 \%$ after a single dose of inclisiran and $35.5 \%$ to $52.6 \%$ after two doses $(p<$ 0.001 for all comparisons vs. placebo). This reduction in LDL cholesterol is similar to that achieved with PCSK9 monoclonal antibodies (evolocumab and alirocumab). At day 240, PCSK9 and LDL cholesterol levels remained significantly lower than the baseline levels in association with all inclisiran doses. Inclisiran might have a potential advantage regarding the injection burden of two or three times a year compared with the PCSK9 monoclonal antibodies that require injections every 2 weeks. A phase III cardiovascular outcomes trial, A Randomized Trial Assessing the Effects of Inclisiran on Clinical Outcomes Among People With Cardiovascular Disease (ORION-4, NCT03705234), is currently ongoing.

The PCSK9 monoclonal antibodies efficiently inhibit PCSK9 resulting in markedly low plasma LDL cholesterol. However, this passive immunization approach requires frequent injections because of the relatively short half-life of the monoclonal antibodies. Therefore, active immunization could provide an efficient and durable therapeutic approach for hypercholesterolemia. Recently, several preclinical studies have revealed promising results in producing long-term anti-PCSK9 antibodies by vaccination $[13,82-84]$. Most recently, Momtazi-Borojeni et al. [84] evaluated the effect of a nanoliposomal PCSK9-specific active vaccine in producing long-lasting anti-PCSK9 antibodies in a BALB/c mice model. They demonstrated that the vaccine improved the high immunoglobulin G antibody response against the PCSK9 peptide, resulting in markedly decreased plasma levels of PCSK9 by $52.5 \%$ compared with the control group at 2 weeks. The vaccine was able to stimulate a long-lasting immune response against $\mathrm{PCSK}_{9}$ and acquired a maximum titer eight weeks after the primary vaccination. The level of plasma anti-PCSK9 antibodies was found to drop with an in vivo half-life of around 5 months. One of the anti-PCSK9 vaccines, ATo4A, has already undergone a phase I clinical trial (NCTo2508896) [13].

\section{EXPERT OPINION ON THE USE OF PCSK IN- HIBITORS}

Two pivotal RCTs, the FOURIER and ODYSSEY OUTCOMES trials, had different strategies to use anti-PC$\mathrm{SK}_{9}$ antibodies for LDL cholesterol reduction in patients with ASCVD $[9,10]$. In the FOURIER trial, patients were randomly assigned in a 1:1 ratio to receive subcutaneous injections of evolocumab (either $140 \mathrm{mg}$ every 2 weeks or $420 \mathrm{mg}$ monthly) or the placebo. Evolocumab significantly reduced the risk of the primary endpoint (a composite of cardiovascular death, myocardial infarction, stroke, hospitalization for unstable angina, or coronary revascularization), but there was no difference in cardiovascular mortality or all-cause mortality between the two groups. In the ODYSSEY OUTCOMES trial, patients were randomly assigned in a 1:1 ratio to receive alirocumab subcutaneously at a dose of $75 \mathrm{mg}$ or the placebo every 2 weeks, and the dose of alirocumab was adjusted in a blinded fashion to target an LDL cholesterol level of 25 to $50 \mathrm{mg} / \mathrm{dL}$ ( 0.6 to $1.3 \mathrm{mmol} / \mathrm{L}$ ). Alirocumab significantly reduced the primary endpoint events (a composite of death from coronary heart disease, non-fatal myocardial infarction, fatal or non-fatal ischemic stroke, or unstable angina requiring hospitalization) and all-cause mortality. Given the unknown long-term safety and high cost of anti-PCSK9 antibodies, the strategy of adjusting the dose of evolocumab or alirocumab to target an LDL cholesterol level of 25 to $50 \mathrm{mg} / \mathrm{dL}$ ( 0.6 to $1.3 \mathrm{mmol} / \mathrm{L}$ ) would be optimal in the current scenario.

A recent retrospective study analyzed 6,151 alirocumab or evolocumab new users from 2015 to 2017 in the USA and demonstrated that $52.2 \%$ of the patients had an interruption in PCSK9 inhibitor therapy in the first year after treatment initiation [85]. Furthermore, after PCSK9 initiation, many patients discontinue or down-titrate other LDL-lowering agents such as statins or ezetimibe. These findings suggest that many new PCSK9 inhibitor users may remain at high risk of cardiovascular events because of interruptions or discontinuations in LDL-lowering therapy. However, there is a paucity of data regarding the reasons why a PCSK9 inhibitor or other LDL-lowering therapy was interrupted. Therefore, further study is warranted to provide further insights into the challenges physicians or patients face in achieving guideline-recommended LDL cholesterol levels. 


\section{CONCLUSIONS}

Two PCSK9 monoclonal antibodies evolocumab and alirocumab provide additional LDL cholesterol reduction and reduce cardiovascular events on top of background statin therapy in patients with ASCVD. From the available data, the safety profiles of evolocumab and alirocumab are highly favorable, although their long-term safety remains undetermined. Because the high cost of PCSK9 monoclonal antibodies is limiting their widespread use at present, their price needs to be reduced considerably to make them cost-effective. More comparative data on the efficacy and safety of PCSK9 inhibitors according to ethnicity is needed. Current guidelines for the management of dyslipidemia recommend the use of PCSK9 inhibitors in patients at high or very high risk of future cardiovascular events, in whom LDL cholesterol targets are not achieved with maximally tolerated statins and ezetimibe, and in those who are statin intolerant. Further cardiovascular outcomes trials with PCSK9 monoclonal antibodies in primary prevention and in the early phase of acute cardiovascular events are needed. Future studies are required to assess the role of alternative approaches to PCSK9 inhibition, such as the use of siRNAs and vaccination.

\section{Conflict of interest}

No potential conflict of interest relevant to this article was reported.

\section{Acknowledgments}

This study was supported by the Bio and Medical Technology Development Program of the National Research Foundation (NRF) and funded by the Korean government (MSIT) (2018M3A9E2024584).

\section{REFERENCES}

1. GBD 2016 Mortality Collaborators. Global, regional, and national under-5 mortality, adult mortality, age-specific mortality, and life expectancy, 1970-2016: a systematic analysis for the Global Burden of Disease Study 2016. Lancet 2017;390:1084-1150

2. Mathers CD, Loncar D. Projections of global mortality and burden of disease from 2002 to 2030. PLoS Med 2006;3:e442.

3. Ference BA, Ginsberg HN, Graham I, et al. Low-density lipoproteins cause atherosclerotic cardiovascular disease. 1. Evidence from genetic, epidemiologic, and clinical studies: a consensus statement from the European Atherosclerosis Society Consensus Panel. Eur Heart J 2017;38:2459-2472.

4. Cholesterol Treatment Trialists' (CTT) Collaborators, Mihaylova B, Emberson J, et al. The effects of lowering LDL cholesterol with statin therapy in people at low risk of vascular disease: meta-analysis of individual data from 27 randomised trials. Lancet 2012;380:581-590.

5. Jernberg T, Hasvold P, Henriksson M, Hjelm H, Thuresson M, Janzon M. Cardiovascular risk in post-myocardial infarction patients: nationwide real world data demonstrate the importance of a long-term perspective. Eur Heart J 2015;36:1163-1170.

6. Thompson PD, Panza G, Zaleski A, Taylor B. Statin-associated side effects. J Am Coll Cardiol 2016;67:2395-2410.

7. Abifadel M, Varret M, Rabes JP, et al. Mutations in PCSK9 cause autosomal dominant hypercholesterolemia. Nat Genet 2003;34:154-156.

8. Cohen JC, Boerwinkle E, Mosley TH Jr, Hobbs HH. Sequence variations in PCSK9, low LDL, and protection against coronary heart disease. N Engl J Med 2006;354:1264-1272.

9. Sabatine MS, Giugliano RP, Keech AC, et al. Evolocumab and clinical outcomes in patients with cardiovascular disease. N Engl J Med 2017;376:1713-1722.

10. Schwartz GG, Steg PG, Szarek M, et al. Alirocumab and cardiovascular outcomes after acute coronary syndrome. N Engl J Med 2018;379:2097-2107.

11. Taylor BA, Thompson PD. Statins and their effect on PCSK9-impact and clinical relevance. Curr Atheroscler Rep 2016;18:46.

12. Shapiro MD, Fazio S. From lipids to inflammation: new approaches to reducing atherosclerotic risk. Circ Res 2016;118:732-749.

13. Landlinger C, Pouwer MG, Juno C, et al. The ATo4A vaccine against proprotein convertase subtilisin/kexin type 9 reduces total cholesterol, vascular inflammation, and atherosclerosis in $\mathrm{APOE}^{\star}{ }_{3}$ Leiden.CETP mice. Eur Heart J 2017;38:2499-2507.

14. Blom DJ, Hala T, Bolognese M, et al. A 52-week placebo-controlled trial of evolocumab in hyperlipidemia. $\mathrm{N}$ Engl J Med 2014;370:1809-1819. 
15. Koren MJ, Lundqvist P, Bolognese M, et al. Anti-PCSK9 monotherapy for hypercholesterolemia: the MENDEL-2 randomized, controlled phase III clinical trial of evolocumab. J Am Coll Cardiol 2014;63:2531-2540.

16. Robinson JG, Nedergaard BS, Rogers WJ, et al. Effect of evolocumab or ezetimibe added to moderate- or high-intensity statin therapy on LDL-C lowering in patients with hypercholesterolemia: the LAPLACE-2 randomized clinical trial. JAMA 2014;311:1870-1882.

17. Stroes E, Colquhoun D, Sullivan D, et al. Anti-PCSK9 antibody effectively lowers cholesterol in patients with statin intolerance: the GAUSS-2 randomized, placebo-controlled phase 3 clinical trial of evolocumab. J Am Coll Cardiol 2014;63:2541-2548.

18. Raal FJ, Honarpour N, Blom DJ, et al. Inhibition of PCSK9 with evolocumab in homozygous familial hypercholesterolaemia (TESLA Part B): a randomised, double-blind, placebo-controlled trial. Lancet 2015;385:341-350.

19. Raal FJ, Stein EA, Dufour R, et al. PCSK9 inhibition with evolocumab (AMG 145) in heterozygous familial hypercholesterolaemia (RUTHERFORD-2): a randomised, double-blind, placebo-controlled trial. Lancet 2015;385:331-340.

20. Sabatine MS, Giugliano RP, Wiviott SD, et al. Efficacy and safety of evolocumab in reducing lipids and cardiovascular events. N Engl J Med 2015;372:1500-1509.

21. Kiyosue A, Honarpour N, Kurtz C, Xue A, Wasserman SM, Hirayama A. A phase 3 study of evolocumab (AMG 145) in statin-treated japanese patients at high cardiovascular risk. Am J Cardiol 2016;117:40-47.

22. Nissen SE, Stroes E, Dent-Acosta RE, et al. Efficacy and tolerability of evolocumab vs ezetimibe in patients with muscle-related statin intolerance: the GAUSS-3 randomized clinical trial. JAMA 2016;315:1580-1590.

23. Lagace TA, Curtis DE, Garuti R, et al. Secreted PCSK9 decreases the number of LDL receptors in hepatocytes and in livers of parabiotic mice. J Clin Invest 2006;116:2995-3005.

24. Bays H, Gaudet D, Weiss R, et al. Alirocumab as add-on to atorvastatin versus other lipid treatment strategies: ODYSSEY OPTIONS I randomized trial. J Clin Endocrinol Metab 2015;100:3140-3148.

25. Kastelein JJ, Ginsberg HN, Langslet G, et al. ODYSSEY FH I and FH II: 78 week results with alirocumab treatment in 735 patients with heterozygous familial hypercholesterolaemia. Eur Heart J 2015;36:2996-3003.

26. Kereiakes DJ, Robinson JG, Cannon CP, et al. Efficacy and safety of the proprotein convertase subtilisin/kexin type
9 inhibitor alirocumab among high cardiovascular risk patients on maximally tolerated statin therapy: the ODYSSEY COMBO I study. Am Heart J 2015;169:906-915.

27. Robinson JG, Farnier M, Krempf M, et al. Efficacy and safety of alirocumab in reducing lipids and cardiovascular events. N Engl J Med 2015;372:1489-1499.

28. Roth EM, McKenney JM. ODYSSEY MONO: effect of alirocumab $75 \mathrm{mg}$ subcutaneously every 2 weeks as monotherapy versus ezetimibe over 24 weeks. Future Cardiol 2015;11:27-37.

29. Farnier M, Jones P, Severance R, et al. Efficacy and safety of adding alirocumab to rosuvastatin versus adding ezetimibe or doubling the rosuvastatin dose in high cardiovascular-risk patients: the ODYSSEY OPTIONS II randomized trial. Atherosclerosis 2016;244:138-146.

30. Ginsberg HN, Rader DJ, Raal FJ, et al. Efficacy and safety of alirocumab in patients with heterozygous familial hypercholesterolemia and LDL-C of $160 \mathrm{mg} / \mathrm{dl}$ or higher. Cardiovasc Drugs Ther 2016;30:473-483.

31. Roth EM, Moriarty PM, Bergeron J, et al. A phase III randomized trial evaluating alirocumab $300 \mathrm{mg}$ every 4 weeks as monotherapy or add-on to statin: ODYSSEY CHOICE I. Atherosclerosis 2016;254:254-262.

32. Stroes E, Guyton JR, Lepor N, et al. Efficacy and safety of alirocumab $150 \mathrm{mg}$ every 4 weeks in patients with hypercholesterolemia not on statin therapy: the ODYSSEY CHOICE II study. J Am Heart Assoc 2016;5:e003421.

33. El Shahawy M, Cannon CP, Blom DJ, et al. Efficacy and safety of alirocumab versus ezetimibe over 2 years (from ODYSSEY COMBO II). Am J Cardiol 2017;120:931-939.

34. Qamar A, Giugliano RP, Keech AC, et al. Interindividual variation in low-density lipoprotein cholesterol level reduction with evolocumab: an analysis of FOURIER Trial Data. JAMA Cardiol 2019;4:59-63.

35. Karatasakis A, Danek BA, Karacsonyi J, et al. Effect of PCSK, inhibitors on clinical outcomes in patients with hypercholesterolemia: a meta-analysis of 35 randomized controlled trials. J Am Heart Assoc 2017;6:eoo6910.

36. Wiviott SD, Giugliano RP, Morrow DA, et al. Effect of evolocumab on type and size of subsequent myocardial infarction: a prespecified analysis of the FOURIER randomized clinical trial. JAMA Cardiol 2020;5:787-793.

37. Giugliano RP, Pedersen TR, Saver JL, et al. Stroke prevention with the $\mathrm{PCSK}_{9}$ (proprotein convertase subtilisin-kexin type 9) inhibitor evolocumab added to statin in high-risk patients with stable atherosclerosis. Stroke 
2020;51:1546-1554.

38. Jukema JW, Zijlstra LE, Bhatt DL, et al. Effect of alirocumab on stroke in ODYSSEY OUTCOMES. Circulation 2019;140:2054-2062.

39. Du H, Li X, Su N, et al. Proprotein convertase subtilisin/kexin 9 inhibitors in reducing cardiovascular outcomes: a systematic review and meta-analysis. Heart 2019;105:1149-1159.

40. Gencer B, Kronenberg F, Stroes ES, Mach F. Lipoprotein(a): the revenant. Eur Heart J 2017;38:1553-1560.

41. Willeit P, Ridker PM, Nestel PJ, et al. Baseline and on-statin treatment lipoprotein(a) levels for prediction of cardiovascular events: individual patient-data meta-analysis of statin outcome trials. Lancet 2018;392:1311-1320.

42. Cao YX, Liu HH, Li S, Li JJ. A meta-analysis of the effect of PCSK9-monoclonal antibodies on circulating lipoprotein (a) levels. Am J Cardiovasc Drugs 2019;19:87-97.

43. O’Donoghue ML, Fazio S, Giugliano RP, et al. Lipoprotein(a), $\mathrm{PCSK}_{9}$ inhibition, and cardiovascular risk. Circulation 2019;139:1483-1492.

44. Reyes-Soffer G, Pavlyha M, Ngai C, et al. Effects of PCSK, inhibition with alirocumab on lipoprotein metabolism in healthy humans. Circulation 2017;135:352-362.

45. Watts GF, Chan DC, Somaratne R, et al. Controlled study of the effect of proprotein convertase subtilisin-kexin type 9 inhibition with evolocumab on lipoprotein(a) particle kinetics. Eur Heart J 2018;39:2577-2585.

46. Edmiston JB, Brooks N, Tavori H, et al. Discordant response of low-density lipoprotein cholesterol and lipoprotein(a) levels to monoclonal antibodies targeting proprotein convertase subtilisin/kexin type 9. J Clin Lipidol 2017;11:667-673.

47. Shapiro MD, Minnier J, Tavori H, et al. Relationship between low-density lipoprotein cholesterol and lipoprotein(a) lowering in response to PCSK9 inhibition with evolocumab. J Am Heart Assoc 2019;8:e010932.

48. Tang Z, Jiang L, Peng J, et al. PCSK9 siRNA suppresses the inflammatory response induced by oxLDL through inhibition of NF- $\mathrm{B}$ activation in THP-1-derived macrophages. Int J Mol Med 2012;30:931-938.

49. Ferri N, Tibolla G, Pirillo A, et al. Proprotein convertase subtilisin kexin type 9 (PCSK9) secreted by cultured smooth muscle cells reduces macrophages LDLR levels. Atherosclerosis 2012;220:381-386.

50. Ding Z, Liu S, Wang X, et al. Hemodynamic shear stress via ROS modulates PCSK9 expression in human vascular endothelial and smooth muscle cells and along the mouse aorta. Antioxid Redox Signal 2015;22:760-771.

51. Ding Z, Liu S, Wang X, et al. Cross-talk between LOX-1 and PCSK9 in vascular tissues. Cardiovasc Res 2015;107:556-567.

52. Tang ZH, Peng J, Ren Z, et al. New role of PCSK9 in atherosclerotic inflammation promotion involving the TLR4/NF-кB pathway. Atherosclerosis 2017;262:113-122.

53. Kuhnast S, van der Hoorn JW, Pieterman EJ, et al. Alirocumab inhibits atherosclerosis, improves the plaque morphology, and enhances the effects of a statin. J Lipid Res 2014;55:2103-2112.

54. Muldoon MF, Barger SD, Ryan CM, et al. Effects of lovastatin on cognitive function and psychological well-being. Am J Med 2000;108:538-546.

55. Muldoon MF, Ryan CM, Sereika SM, Flory JD, Manuck SB. Randomized trial of the effects of simvastatin on cognitive functioning in hypercholesterolemic adults. Am J Med 2004;117:823-829.

56. Navarese EP, Kolodziejczak M, Schulze V, et al. Effects of proprotein convertase subtilisin/kexin type 9 antibodies in adults with hypercholesterolemia: a systematic review and meta-analysis. Ann Intern Med 2015;163:40-51.

57. Toth PP, Descamps O, Genest J, et al. Pooled safety analysis of evolocumab in over 6000 patients from double-blind and open-label extension studies. Circulation 2017;135:1819-1831.

58. Giugliano RP, Mach F, Zavitz K, et al. Cognitive function in a randomized trial of evolocumab. N Engl J Med 2017;377:633-643.

59. Harvey PD, Sabbagh MN, Harrison JE, et al. No evidence of neurocognitive adverse events associated with alirocumab treatment in 3340 patients from 14 randomized phase 2 and 3 controlled trials: a meta-analysis of individual patient data. Eur Heart J 2018;39:374-381.

6o. Foltz IN, Karow M, Wasserman SM. Evolution and emergence of therapeutic monoclonal antibodies: what cardiologists need to know. Circulation 2013;127:2222-2230.

61. Ridker PM, Tardif JC, Amarenco P, et al. Lipid-reduction variability and antidrug-antibody formation with bococizumab. N Engl J Med 2017;376:1517-1526.

62. Koren MJ, Sabatine MS, Giugliano RP, et al. Long-term low-density lipoprotein cholesterol-lowering efficacy, persistence, and safety of evolocumab in treatment of hypercholesterolemia: results up to 4 years from the open-label OSLER-1 extension study. JAMA Cardiol 2017;2:598-607.

63. Farnier M, Hovingh GK, Langslet G, et al. Long-term 
safety and efficacy of alirocumab in patients with heterozygous familial hypercholesterolemia: an open-label extension of the ODYSSEY program. Atherosclerosis 2018;278:307-314.

64. Blom DJ, Djedjos CS, Monsalvo ML, et al. Effects of evolocumab on vitamin e and steroid hormone levels: results from the 52-week, phase 3, double-blind, randomized, placebo-controlled DESCARTES study. Circ Res 2015;117:731-741.

65. Arrieta A, Hong JC, Khera R, Virani SS, Krumholz HM, Nasir K. Updated cost-effectiveness assessments of PCSK9 inhibitors from the perspectives of the health system and private payers: insights derived from the FOURIER trial. JAMA Cardiol 2017;2:1369-1374.

66. Kazi DS, Penko J, Coxson PG, Guzman D, Wei PC, Bibbins-Domingo K. Cost-effectiveness of alirocumab: a just-in-time analysis based on the ODYSSEY outcomes trial. Ann Intern Med 2019;170:221-229.

67. Kaufman TM, Warden BA, Minnier J, et al. Application of PCSK9 inhibitors in practice. Circ Res 2019;124:32-37.

68. Knickelbine T, Jia L, White SK, et al. A systematic approach for successful $\mathrm{PCSK}_{9}$ inhibitor prescribing in clinical practice. J Clin Lipidol 2019;13:265-271.

69. Reynolds VW, Chinn ME, Jolly JA, et al. Integrated specialty pharmacy yields high PCSK9 inhibitor access and initiation rates. J Clin Lipidol 2019;13:254-264.

70. Grundy SM, Stone NJ, Bailey AL, et al. 2018 AHA/ACC/ AACVPR/AAPA/ABC/ACPM/ADA/AGS/APhA/ASPC/NLA/ PCNA guideline on the management of blood cholesterol: a report of the American College of Cardiology/American Heart Association task force on clinical practice guidelines. Circulation 2019;139:e1082-e1143.

71. Birmingham BK, Bujac SR, Elsby R, et al. Rosuvastatin pharmacokinetics and pharmacogenetics in Caucasian and Asian subjects residing in the United States. Eur J Clin Pharmacol 2015;71:329-340.

72. Li YF, Feng QZ, Gao WQ, Zhang XJ, Huang Y, Chen YD. The difference between Asian and Western in the effect of LDL-C lowering therapy on coronary atherosclerotic plaque: a meta-analysis report. BMC Cardiovasc Disord 2015;15:6.

73. Wang C, Zheng Q, Zhang M, Lu H. Lack of ethnic differences in the pharmacokinetics and pharmacodynamics of evolocumab between Caucasian and Asian populations. Br J Clin Pharmacol 2019;85:114-125.
74. Han Y, Chen J, Chopra VK, et al. ODYSSEY EAST: alirocumab efficacy and safety vs ezetimibe in high cardiovascular risk patients with hypercholesterolemia and on maximally tolerated statin in China, India, and Thailand. J Clin Lipidol 2020;14:98-108.

75. Koh KK, Nam CW, Chao TH, et al. A randomized trial evaluating the efficacy and safety of alirocumab in South Korea and Taiwan (ODYSSEY KT). J Clin Lipidol 2018;12:162-172.

76. Rhee EJ, Kim HC, Kim JH, et al. 2018 Guidelines for the management of dyslipidemia. Korean J Intern Med 2019;34:723-771.

77. Mach F, Baigent C, Catapano AL, et al. 2019 ESC/EAS guidelines for the management of dyslipidaemias: lipid modification to reduce cardiovascular risk. Eur Heart J 2020;41:111-188.

78. Schwartz GG, Olsson AG, Ezekowitz MD, et al. Effects of atorvastatin on early recurrent ischemic events in acute coronary syndromes: the MIRACL study: a randomized controlled trial. JAMA 2001;285:1711-1718.

79. Cannon CP, Braunwald E, McCabe CH, et al. Intensive versus moderate lipid lowering with statins after acute coronary syndromes. N Engl J Med 2004;350:1495-1504.

8o. Koskinas KC, Windecker S, Pedrazzini G, et al. Evolocum$\mathrm{ab}$ for early reduction of LDL cholesterol levels in patients with acute coronary syndromes (EVOPACS). J Am Coll Cardiol 2019;74:2452-2462.

81. Ray KK, Landmesser U, Leiter LA, et al. Inclisiran in patients at high cardiovascular risk with elevated LDL cholesterol. N Engl J Med 2017;376:1430-1440.

82. Pan Y, Zhou Y, Wu H, et al. A therapeutic peptide vaccine against PCSK9. Sci Rep 2017;7:12534.

83. Kawakami R, Nozato Y, Nakagami H, et al. Development of vaccine for dyslipidemia targeted to a proprotein convertase subtilisin/kexin type 9 (PCSK9) epitope in mice. PLoS One 2018;13:e0191895.

84. Momtazi-Borojeni AA, Jaafari MR, Badiee A, Sahebkar A. Long-term generation of antiPCSK9 antibody using a nanoliposome-based vaccine delivery system. Atherosclerosis 2019;283:69-78.

85. Rymer JA, Mues KE, Monda KL, et al. Use of low-density lipoprotein-lowering therapies before and after PCSK9 inhibitor initiation. J Am Heart Assoc 2020;9:e014347. 